\title{
Conditions et limites d'une démarche de prospective métier dans un secteur de PME touché par la crise économique
}

\author{
par Céline Remy ${ }^{81}$ et François Pichault ${ }^{82}$
}

\begin{abstract}
Résumé
Cet article vise à rendre compte de la difficulté de mener un exercice prospectif dans un secteur composé de petites entreprises et touché par une crise économique. II se structure en trois parties : la première consiste en la mise en perspective des controverses du champ de la méthode prospective. La deuxième partie se dédie à une étude de cas pour laquelle nous avons recouru à la méthode prospective dans un secteur d'activité regroupant fabrications métalliques et industrie technologique. La troisième partie discute des différents aspects méthodologiques issus de l'intervention et tire des enseignements.
\end{abstract}

\section{Abstract}

This article aims to show the difficulty of conducting a prospective exercise in a sector composed of small companies and affected by one economic crisis. It is divided into three parts: the first one puts into perspective the controversies in the field of the prospective method. The second part is dedicated to a case study where we used the prospective method in a sector of metal manufactures and technology industry. The third part discusses various methodological issues encountered in the intervention and draws lessons.

Cet article vise à rendre compte de la difficulté de mener un exercice de prospective dans un secteur composé de petites entreprises et touché par une crise économique. Le secteur étudié se constitue de manière paritaire ${ }^{83}$, avec des partenaires multiples (entreprises, organismes de formation, fonds de formation). II nous a semblé nécessaire, dans un premier temps, de faire état des controverses qui animent le champ de la prospective en général, et de la prospective métier, en particulier. Nous présentons, dans un deuxième temps, une étude de cas où nous avons recouru à la méthode prospective dans un secteur d'activité ${ }^{84}$ regroupant fabrications métalliques et industrie technologique. Cette

\footnotetext{
81. CÉLINe Remy, Chargée de recherche, Université de Liège, LENTIC, CRIS, celine.remy@ulg.ac.be

82. Françols Pichault, Professeur HEC-Ecole de gestion, Université de Liège, LENTIC, f.pichault@ulg.ac.be

83. En Belgique, un secteur paritaire désigne le fait d'avoir à nombre équivalent les syndicats et les patrons.

84. Le secteur Agoria rassemble 1580 entreprises et quelque 280.000 travailleurs pour toute la Belgique. Sa mission principale est de veiller à l'instauration d'un environnement socio-économique optimal, propice à ses membres - et à leurs travailleurs - et de les assister au mieux dans toutes les étapes de leur développement. II regroupe différentes activités : aérospatiale, automatisation industrielle, automobile, contracting \& maintenance, électrotechnique, mécanique \& mécatronique, métaux et matériaux, montage \& grues, plastiques et composites, produits de construction, sécurité et défense, technologies de l'information et de la communication, transformation du métal. L'enquête a visé l'association régionale des provinces de Liège et du Luxembourg.
} 


\section{9

partie est articulée autour des difficultés rencontrées lors de l'intervention : 1) une demande et des modalités d'intervention à préciser, 2) des sources d'information à fiabiliser, 3) une projection dans l'avenir à stimuler et 4) un discours généraliste à éviter. Dans un troisième temps, nous menons une discussion sur ces différents aspects méthodologiques pour en tirer les principaux enseignements.

\section{L'enjeu d'une vision prospective sur les compétences : état des controverses}

La prospective appliquée aux organisations semble être apparue au début du $20^{\mathrm{ème}}$ siècle. Elle est introduite en France, fin des années cinquante, par Gaston Berger. Il semblerait que les bases de la discipline aient été posées par le romancier H.-G. Wells qui propose de « connaître les faits de l'avenir » (Scouarnec, 2008, p.3). Selon Berger (1964), la prospective est d'abord une attitude avant d'être une méthode ou une discipline. Cette attitude " prospective » repose sur cinq principes : " voir loin, voir large, analyser en profondeur, prendre des risques, penser à l'homme »(Berger, 1964, p.271-275).

La prospective peut se définir soit de manière brève et concise, comme « l'étude de l'avenir lointain »(Berger, 1964, p.271), soit de manière approfondie et détaillée, comme « une synthèse du temps passé, présent et futur, des multi-dimensions de la société, des lois de la nature, de rapports intentionnels et inintentionnels, des contraintes objectives des héritages et des rythmes, de l'inertie et du dynamisme des forces sociales, des projets d'acteurs dotés d'inspiration, de stratégies et de moyens »(Gonod 1996, p.6). Une autre définition se situant à mi-chemin entre les deux premières permet de cerner rapidement ce que recouvre la technique de prospective. En référence au guide sur la prospective de Jouvenel (2009, p.3) « faire de la prospective consiste à explorer quels sont les futurs possibles en germe dans la situation actuelle, pour aider à la détermination des futurs souhaitables et à l'identification des moyens à mettre en œuvre pour les atteindre ».

La méthode prospective a, progressivement, donné naissance à une méthode spécifique dénommée : " prospective métier ». Cette dernière émerge à la suite d'une revalorisation du concept de métier. Face à ce glissement de la logique de poste à la logique de compétence dans les années 80-90 et aux problématiques contemporaines de l'identité au travail qui y sont liées, la prospective métier semble être plus à même de favoriser une gestion des ressources humaines innovantes.

De ce fait, elle est en mesure de tenir compte des évolutions internes et externes de l'organisation, tout en les inscrivant dans le temps et dans l'espace. Elle permet tant au travailleur de prendre conscience de ses compétences et de 


\section{Conditions et limites d'une démarche de prospective métier dans un secteur de PME touché par la crise économique}

leurs possibles évolutions dans le temps (Thamain, 2009) qu'à l'entreprise de gérer ses besoins en compétence. La prospective métier peut être considérée comme la « capacité de détection précoce de signaux faibles des changements à venir » (Scouarnec et Yanat, 2003, p.2292). Elle est également " un regard sur les avenirs possibles destiné à éclairer l'action présente " (Godet, 1992 in Noguera et Lartigau, 2009, p.298).

Les entreprises sont confrontées à l'évolution constante des métiers. La gestion du quotidien (délais, pannes, pics de production, etc) ne favorise pas nécessairement le développement des capacités de détection de ces évolutions. Face au déficit d'anticipation des entreprises, un dispositif dénommé PGPEC (Plan de Gestion Prévisionnelle de l'Emploi et des Compétences) a été lancé, en France, en 2003 et a été revu en 2007. L'objectif de ce dispositif est « d'inciter les entreprises à entreprendre une action prospective pour faire évoluer les emplois afin de les pérenniser au mieux » (Bellini, 2009, p.1). Contrairement à son " ancêtre » (la gestion prévisionnelle des effectifs), la GPEC se traduit en termes quantitatif et qualitatif.

II semble opportun de se demander si les concepts de "prévision " et de " prospective » recouvrent les mêmes " réalités ». Ces concepts relèvent tous deux d'un besoin d'anticipation par rapport à l'avenir. Cependant, en référence à Thamain (2009), la méthode de " prévision " s'ancre dans une logique dite de " continuité » car elle établit des prévisions basées sur des tendances lourdes, à court terme. Elle repose sur " l'extrapolation du passé » (de Jouvenel H., 1999, p.49). La démarche de prospective, quant à elle, s'inscrit dans une logique de " rupture » par le fait d'avoir pour intention d'identifier des ruptures, des discontinuités dans les modèles connus. Dès lors, elle ne se base pas sur une donnée déjà présente mais elle émerge d'un « construit » (Thamain, 2009, p.274), comme « une chose à faire » (Berger, 1967, p.33).

Les concepts de prévision et de prospective introduisent également chacun la notion de temps dans la gestion (Gilbert, 2006). II semble, néanmoins, qu'une petite nuance est à préciser, en ce sens où, selon Scouarnec et Yanat (2003, p.5), la prospective fait référence à « l'ordre du temps humain » et ce, contrairement à la prévision qui, elle, fait appel à " l'ordre du temps de la physique ». Elle permet, en conséquence, un dépassement des méthodes classiques de gestion prévisionnelle en gestion des ressources humaines qui semblent avoir prouvé leur inadéquation à un environnement concurrentiel de plus en plus global et changeant (Boyer et Scouarnec, 2008). Contrairement à la démarche de prévision, la prospective accorde une importance à la « dimension temporelle « humaine » et à ses caractéristiques d'hétérogénéité, de systémique, de globalité et d'articulation passé/présent/futur » (Scouarnec et Yanat, 2003, p.2295). 


\section{9 \\ management \\ \& avenir}

La méthodologie de la démarche prospective comprend, selon Scouarnec et Boyer (2002), plusieurs étapes : 1) l'appréhension contextuelle, 2) la préformalisation, 3) la construction et 4) la validation. L'appréhension contextuelle représente la phase exploratoire en ce sens où le chercheur s'approprie le sujet d'étude et rencontre une série d'acteurs-experts dans le cadre d'entretiens semidirectifs. La pré-formalisation correspond à l'analyse du contenu des entretiens permettant la construction d'un questionnaire envoyé par la suite aux acteursexperts. L'étape de la construction se réfère à la réalisation d'une analyse du contenu des entretiens en groupe de travail. La validation intègre les résultats des groupes de travail pour proposer un modèle général qui fera l'objet d'une validation par les différents groupes.

La prospective a recours à la méthode des scénarii qui permet d'envisager les futurs possibles (les scénarii) « en se fondant sur un diagnostic en dynamique du territoire et de ses relations avec son environnement » (de Jouvenel, 2009, p.8). La construction de scénarii a été développée par Herman Kahn dans les années 50, aux Etats-Unis. Servant d'aide à la prise de décision, ces représentations imagées donnent un rapide aperçu des différentes voies possibles pour l'avenir. Toujours selon de Jouvenel (2009, p.9), la méthode des scénarii convient parfaitement pour clarifier « les enjeux majeurs et les leviers d'actions dans des situations complexes dont l'avenir paraît difficile à cerner ». Elle peut être définie comme « une démarche synthétique qui, d'une part, simule, étape par étape et d'une manière plausible et cohérente, une suite d'événements conduisant un système à une situation future, et qui, d'autre part, présente une image d'ensemble de celle-ci » (Julien et al., 1975, p.254).

Divers types de scénarii existent : les scénarii exploratoires et les scénarii d'anticipation. Les premiers se basent sur « une situation présente et des tendances qui y prévalent, une suite d'événements conduisant d'une façon logique (nécessaire) à un futur possible. [Tandis que les deuxièmes] décrivent l'image d'un futur possible et souhaitable, décrite par un ensemble donné d'objectifs à réaliser » (Julien et al., 1975, p.254, 256). Les premiers vont du présent vers le futur alors que les deuxièmes vont du futur au présent.

Une des critiques faites à la méthode prospective se réfère à son manque d'opérationnalisation. Face à cette critique, Scouarnec (2008) propose d'entrer dans une nouvelle ère de la prospective offrant des méthodes et des outils mieux adaptés aux pratiques managériales. Deux périodes ont précédé celle-ci : la première regroupe les "fondateurs " de la discipline tandis que la deuxième associe les « méthodologues » qui ont développé la réflexion méthodologique de la démarche. Dans ce troisième mouvement - la vague des « constructeurs »-, Scouarnec (2008) soutient le développement d'une nouvelle perspective normative considérant alors la prospective comme un " savoir actionnable " (Argyris, 1995). 


\section{Conditions et limites d'une démarche de prospective métier dans un secteur de PME touché par la crise économique}

Ainsi, « il est possible de développer un savoir, à la fois valable et pouvant être « mis en action » dans la vie quotidienne, savoir dont l'utilisation par les praticiens ou les chercheurs offre précisément l'occasion de le tester dans la vie de tous les jours » (Argyris, 1995, p.257). Grâce à ce concept de " savoir actionnable », la prospective permet désormais 1) une adéquation entre les intentions des acteurs et les résultats effectifs, 2) un suivi des effets sur le long terme via des missions de veille et de communication et 3 ) un espace de co-conception de l'action collective (Scouarnec, 2008). Pour s'inscrire dans cette nouvelle dynamique de la méthode prospective, il est indispensable de recourir aux « acteurs-experts " (Boyer et Scouarnec, 2008), individus qui sont touchés par la thématique étudiée.

La rencontre "d'acteurs-experts » favorise la collecte des représentations de chacun. La confrontation entre ces visions est utile à la construction « des possibles »(Sen, 2000), c'est-à-dire des diverses possibilités qui sont envisageables dans un contexte ou une situation donnée. Contrairement à la logique déterministe, la focalisation sur « les possibles » permet à la prospective d'ouvrir le champ de réflexion et d'action. Par exemple, en ce qui concerne la prospective métier, cette ouverture s'effectue, selon Boyer et Scouarnec (2008) en termes de possibilités d'apparition, de transformation ou de disparition de métiers. Cette logique « des possibles ॥ fait appel à la capacité de l'expert « d'être un créateur de sens » (Scouarnec, 2008, p.178).

\section{Une démarche paritaire de prospective métier, dans un secteur de TPE/PME affecté par la crise économique}

Notre centre de recherche a été chargé d'une enquête de prospective par l'un des organes paritaires de formation du secteur de l'industrie technologique en Belgique. Ce secteur, essentiellement composé de TPE/PME ${ }^{85}$, est lourdement affecté par la crise économique de 2009. Patrons et syndicats désirent connaître les tendances à venir du secteur afin de mettre en place les actions et dispositifs nécessaires. Nous allons rendre compte de notre intervention en l'articulant autour de quatre points critiques que nous avons rencontrés dans notre pratique d'intervention : 1) une demande et des modalités d'intervention à préciser, 2) des sources d'information à fiabiliser, 3) une projection dans l'avenir à stimuler et 4) un discours généraliste à éviter.

\subsection{Une demande et des modalités d'intervention à préciser}

L'organe de formation sectoriel, commanditaire de l'étude, entend réaliser une enquête auprès de ses membres pour susciter une réflexion qui les aide à

85. L'acronyme TPE/PME désigne Très Petite Entreprise et Petite ou Moyenne Entreprise. La TPE a un effectif de moins de 10 travailleurs salariés avec un chiffre d'affaires annuel ou le total du bilan n'excédant pas 2 millions d'euros. La Petite entreprise en a entre 10 et 50 salariés avec un chiffre d'affaires annuel ou le total du bilan n'excédant pas 10 millions d'euros. La Moyenne entreprise en a entre 50 et moins de 250 travailleurs dont le chiffre d'affaires annuel n'excède pas 50 millions d'euros ou dont le total du bilan n'excède pas 43 millions d'euros. (Source : Journal officiel de l'Union européenne L63/27 du 28.2.2004). 


\section{9 \\ management \\ or avenir}

dépasser le contexte actuel de crise et les engage dans une dynamique à plus long terme. Le fonds souhaite qu'au terme de l'enquête, une série d'actions à mener soient listées au travers de recommandations. Nous tentons de clarifier au mieux la demande d'étude qui se traduit, au départ, comme une étude générale sur l'avenir du secteur. Après quelques discussions, le processus de clarification aboutit : l'étude vise à cerner les tendances actuelles et futures du secteur d'un point de vue sociodémographique, organisationnel, technologique et environnemental ainsi qu'en termes de modalités d'acquisition des compétences pour les métiers ouvriers. L'horizon de la prospective visée par l'étude est d'une dizaine d'années en ce sens où le répondant projette son entreprise dans les années 2020.

Le nouvel énoncé de l'étude fait explicitement référence à la Gestion Prévisionnelle de l'Emploi et des Compétences (GPEC). Avant d'entamer la construction du questionnaire, il est opportun de s'interroger sur ce que recouvre exactement la GPEC, qui constitue en quelque sorte un mot valise, prenant des significations différentes aux yeux de chaque partie prenante. Ainsi, nous commençons l'étude par une période d'appropriation de la problématique via des lectures et la rencontre de personnes clés provenant aussi bien du banc patronal que syndical.

La phase exploratoire nous permet de repérer de manière précise les attentes des parties prenantes. Les responsables patronaux du fonds sectoriel, organe paritaire, prennent contact avec nous pour la réalisation de l'enquête. D'après eux, l'attente principale des syndicats est de cerner l'évolution démographique du secteur, à savoir le nombre de départs à la retraite pour les dix années à venir. Les entretiens exploratoires ont permis de dépasser et de nuancer ce discours relayé en interrogeant directement la partie syndicale. Les syndicats confirment bien le fait qu'ils ont exprimé au monde patronal leur intérêt par rapport à l'évolution démographique du secteur. Mais leur souci principal concerne le nombre important de travailleurs âgés appelés à quitter le secteur : ils aimeraient obtenir plus de précisions quant aux sous-secteurs et aux métiers les plus susceptibles d'être touchés. L'intérêt du monde syndical porte donc moins sur l'évolution des métiers que sur le transfert de compétences à opérer des travailleurs plus âgés vers les plus jeunes.

La rencontre de ces divers acteurs, grâce à la réalisation d'entretiens semidirectifs, nous permet de préciser les représentations de chacun. Le but est clairement de s'informer sur le fonctionnement actuel du secteur ainsi que sur ses évolutions. Nous voulons, sur base des différents constats énoncés et des visions de chaque répondant, construire des scénarii prospectifs.

La rencontre des acteurs révèle aussi trois enjeux principaux. Le premier est la clarification des évolutions démographiques du secteur : les organisations syndicales souhaitent connaître avec précision le nombre de départs à la retraite et surtout, le profil des entreprises concernées et le type de métiers touchés. 


\section{Conditions et limites d'une démarche de prospective métier dans un secteur de PME touché par la crise économique}

Le deuxième enjeu a trait à l'identification des mutations à opérer au niveau de l'offre de formation. Ce sont clairement les opérateurs sectoriels de formation qui sont ici demandeurs, puisque leur mission est de suivre au quotidien les évolutions technologiques des entreprises, l'utilisation de nouveaux matériaux et le développement de nouveaux processus de fabrication, etc. Les résultats de l'enquête doivent leur permettre de développer et d'adapter au mieux leurs formations à l'évolution attendue des déficits de compétences. Un dernier enjeu, pour les responsables sectoriels cette fois, porte sur les initiatives à prendre pour stimuler des innovations stratégiques et/ou organisationnelles parmi les entreprises membres.

\subsection{Des sources d'information à fiabiliser}

Au commencement de l'enquête, des interrogations portent au niveau des sources d'information à activer afin d'obtenir des informations pertinentes et, en conséquence, des résultats significatifs. Le choix des personnes relais (acteursexperts) s'avère crucial. Différents types d'experts sont dès lors rencontrés en fonction de la phase de l'enquête.

La phase exploratoire, alimentée par des entretiens semi-directifs, sert à saisir la problématique du secteur mais également à construire le questionnaire. Pour sélectionner les experts à interroger, nous listons, d'abord, les institutions touchées par cette problématique. II s'agit des organismes de formation, des entreprises du secteur, des organisations syndicales et des instances impliquées dans le développement économique régional (dans le cadre de pôles de compétitivité soutenus par les pouvoirs publics). Chaque institution est composée d'une multitude d'acteurs dont les fonctions et les statuts diffèrent. Comment procéder au choix des candidats les plus pertinents ? Quel sera l'informateur le plus à même de répondre aux questions sur le secteur et, surtout, de développer une vision de ce que sera le secteur dans dix ans?

Nous recourons à cet égard à un échantillon de convenance, sur la base d'une liste de personnes « de confiance » fournie par le commanditaire. Ce dernier nous indique ainsi les personnes ayant, à ses yeux, la capacité de se projeter dans l'avenir, critère essentiel de participation lorsqu'il s'agit d'une enquête de type prospectif. II intervient d'ailleurs lorsque la prise de contact avec les entreprises s'avère difficile, recourant alors à son réseau de contacts interpersonnels. Une quinzaine de personnes (organismes de formation, managers d'entreprise, responsables syndicaux et représentants sectoriels des pôles de compétitivité) ont été sélectionnées de la sorte.

Pour la phase quantitative, nous procédons à un tirage au sort d'une centaine d'entreprises dans la base de données des affiliés au fonds sectoriel. Notre échantillon est raisonné en fonction des critères suivants : le lieu géographique, 


\section{9

le sous-secteur d'activité et le volume d'emplois ouvrier. Une fois l'entreprise sélectionnée, il convient de déterminer le profil du répondant. Celui-ci doit avoir la capacité de se projeter dans l'avenir. La sélection s'effectue sur la base de l'expertise opérationnelle plutôt que GRH. II nous semble en effet important que les interviewés aient à la fois l'expérience du terrain et la connaissance de l'entreprise. Nous nous sommes orientés vers le directeur technique ou le directeur général en fonction de la taille de l'entreprise mais également selon le degré de connaissance interpersonnelle existant entre le commanditaire et certains individus clés de l'entreprise.

La nécessité de stimuler l'imaginaire des répondants et de les aider à se projeter dans le futur nous a conduit à privilégier l'entretien en face à face. Les questionnaires auto-administrés présentent en effet de gros inconvénients : faible taux de réponse, remplissage du questionnaire avec inattention ou bien par une autre personne que celle stipulée au départ, etc. On ne peut ainsi assurer ni la qualité de la récolte des données ni l'inscription du répondant dans une démarche prospective. Au-delà de sa mission classique d'instaurer un climat de confiance, l'intervieweur a pour rôle principal d'aider l'interviewé à entrer dans la dynamique prospective du questionnaire basée sur la construction de scénarii.

\subsection{Une projection dans l'avenir à stimuler}

On sait combien l'anticipation du futur n'est pas chose aisée pour les répondants. Une manière de procéder est de les mettre en situation, c'est-à-dire d'avoir recours aux scénarii prospectifs. La construction de ces scénarii se fait sur base de la phase exploratoire qui permet de dégager les principales tensions perçues par les acteurs-experts. Ces tensions sont identifiées au niveau des tendances sociodémographiques, technologiques et environnementales mais également organisationnelles des entreprises du secteur ainsi qu'au niveau des modalités de formation. Nous pouvons citer, à titre d'exemple, une tension pour chacune de ces tendances : secteur principalement masculin versus métiers de précision principalement féminins, perception d'un secteur en déclin versus perception d'un secteur porteur au vu du rôle clé des technologies dans la vie quotidienne, disparition des métiers artisanaux liée à la robotisation des entreprises versus pérennité des métiers artisanaux, etc.

Sur base de ces tensions, nous construisons le questionnaire dont la particularité est l'élaboration de divers scénarii prospectifs. Ces derniers sont créés en se référant à une entreprise fictive dénommée "VenVert " ${ }^{86}$. Par exemple, nous testons la tension liée à la disparition ou non des métiers traditionnels du secteur via le scénario suivant : "Nous sommes en 2020. L'entreprise VenVert : A) a robotisé toute sa ligne de production de machines hautement performantes, ce

86. VenVert est une entreprise fictive, emblématique des changements escomptés en 2020 pour le secteur des fabrications métalliques et de l'industrie technologique. Elle est utilisée dans le cadre des scénarii pour permettre aux répondants de projeter et positionner leur propre entreprise dans le futur. 


\section{Conditions et limites d'une démarche de prospective métier dans un secteur de PME touché par la crise économique}

qui a entraîné une disparition des métiers " traditionnels " du secteur (soudeurs, usineurs, tourneurs-fraiseurs, fondeurs, etc) ; B) possède des équipements de pointe en machine et en outillage mais elle souhaite conserver les compétences liées aux métiers traditionnels pour la réalisation des tâches de maintenance et de contrôle qualité ; C) continue à recourir prioritairement aux métiers traditionnels car le savoir-faire et l'expertise des hommes sont restés indispensables pour garantir la qualité et la fiabilité de la production ». Les répondants ont ainsi pour mission de sélectionner le scénario étant le plus en adéquation avec la situation de leur entreprise.

Grâce à ce questionnaire, nous testons également la réceptivité des entreprises au développement de formules innovantes en matière de ressources humaines telles que la mutualisation des travailleurs, les nouvelles formes de congés (crédit-temps, temps partiel, interruption de carrière), la présence plus grande de femmes dans un secteur très masculinisé, la suppression du système barémique traditionnel, etc. Ainsi, pour éprouver la probabilité de déployer des formules comme la mutualisation des travailleurs au sein des entreprises, nous proposons aux acteurs-experts de se positionner par rapport au scénario suivant : « Pour faire face à ce phénomène du vieillissement, l'entreprise VenVert a finalement décidé de mettre en place différentes formules. Elle utilise notamment la mutualisation de travailleurs, grâce à laquelle elle détache ponctuellement ou à temps partiel des travailleurs seniors dans d'autres entreprises de la région. Ainsi, elle peut toujours compter sur leur expertise tout en rentabilisant leur temps de travail au maximum ».

La construction d'un questionnaire sous forme de scénarii prospectifs a une double vocation : d'une part, rendre plus concrète la réponse à des questions sur le futur et, d'autre part, favoriser l'engagement des répondants. Compte tenu de l'exigence de l'exercice proposé, l'entretien en face à face vient évidemment apporter une aide précieuse. II l'est d'autant plus que certaines parties du questionnaire exigent de la part du répondant de s'inscrire dans un mouvement d'aller-retour permanent entre la situation vécue actuellement et la situation future de l'entreprise. Ce type d'exercice représente pour lui une " charge mentale lourde » en ce sens où son esprit et son imaginaire sont fortement sollicités.

\subsection{Un discours généraliste à éviter}

Une partie du questionnaire est centrée sur les métiers du secteur et, plus particulièrement, sur les modalités d'acquisition des compétences pour ceux-ci. Le souhait du commanditaire est de traduire cette interrogation de manière à obtenir une analyse de type statistique dont l'optique est d'éviter des constats trop généraux. Nous concrétisons, ainsi, sous forme de questions fermées, les transformations potentielles des métiers. 


\section{9

Comme nous l'avons stipulé plus haut, la phase exploratoire de l'étude a permis l'identification d'une série de tensions portant notamment sur la formation des ouvriers. L'énumération de ces tensions sert à la construction d'une grille d'analyse basée sur des couples d'oppositions, telles que le lieu de formation (interne versus externe à l'entreprise), le type de parcours à prôner pour la formation des ouvriers (parcours de formation prédéfini versus parcours de formation " taillé sur mesure » en fonction des compétences du travailleur), le contenu de la formation (acquisition de compétences " hard », c'est-à-dire techniques, versus acquisition de compétences "soft », c'est-à-dire le savoir-être), le statut de la formation (sur mesure et spécifique à l'entreprise versus validée et certifiée correspondant aux besoins de diverses entreprises) et la pédagogie à utiliser (formation via l'accompagnement d'un travailleur expérimenté versus formation via un professionnel externe, formation sur des machines réelles versus simulations virtuelles).

Ces tensions font émerger deux types de profil en termes de formation, à savoir la formation de type traditionnel et la formation $"$ capacitante $»^{87}$. Le premier recourt à l'image du jeune apprenti débutant sa carrière dans l'entreprise auprès d'un travailleur expérimenté. La transmission de la connaissance et de l'expérience a lieu dans l'échange informel, en situation de travail. Tandis que le deuxième profil correspond plutôt à une formalisation de l'acquisition des compétences. Le travailleur est, ainsi, amené à suivre des formations de type "soft » dans l'optique d'obtenir des outils pour mieux appréhender le monde du travail et, en conséquence, pour développer sa réflexivité. Ces formations se déroulent généralement à l'extérieur de l'entreprise, en la compagnie d'un professionnel.

Pour chacun des trois métiers principaux de leur entreprise, les répondants spécifient la situation actuelle, probable et souhaitée à travers la grille bipolaire. Au niveau de cette partie du questionnaire, une certaine forme de lassitude s'exprime. Elle donne lieu à des résultats décevants d'un point de vue statistique, les répondants parvenant difficilement à différencier les trois types de situation qui leur sont suggérées.

\section{Discussion}

Sur base de la présentation de notre intervention, nous allons procéder à l'analyse des différentes difficultés rencontrées lors de la réalisation de l'enquête.

\subsection{Une exigence de contextualisation}

Une démarche prospective s'effectue toujours en interaction avec des acteurs qui nouent entre eux des alliances, poursuivent certains enjeux, déploient en

87. Ce terme fait référence au concept de capacitation d'Armatya Kumar Sen, économiste indien. 


\section{Conditions et limites d'une démarche de prospective métier dans un secteur de PME touché par la crise économique}

conséquence des stratégies. II ne s'agit nullement d'un exercice désincarné : il importe donc de le contextualiser.

II faut reconnaître que cette démarche n'a pas été menée à bien. Face à une demande d'intervention difficile à éclaircir, nous clarifions la méthodologie mais nous accordons peu d'importance aux modalités d'intervention. Ce n'est qu'en fin d'enquête, lors d'une réunion de préparation à l'analyse des résultats, que nous nous rendons compte - sans doute trop tard dans le processus - de l'importance de clarifier les modalités de restitution et le message à tenir auprès de toutes les parties prenantes. C'est à ce moment que nous comprenons toute l'importance de cibler nos recommandations en fonction des différents acteurs. On voit ainsi la nécessité d'entrer dans un « processus de contextualisation » (Pichault, 2005) : le cadre n'étant pas clairement posé lors de la demande initiale, cela a renforcé les difficultés de l'exercice.

Nous avons pris conscience a posteriori de la nécessité d'identifier de façon minutieuse les différents destinataires du feed-back de l'enquête. Pichault (2005) souligne l'importance d'inclure les diverses personnes interrogées dans le cadre d'une étude. Même s'il n'est pas aisé de négocier cette question au préalable, une telle pratique fait partie du contrat passé avec les informateurs : " Vous nous aidez dans notre collecte d'informations et nous vous fournissons en retour les résultats de notre démarche » (Pichault, 2005). Agir ainsi favorise les bonnes relations avec les informateurs d'une part, et dynamise les « boucles d'information » (Friedberg, 1993), d'autre part.

Après avoir défini les destinataires, il paraît essentiel de cerner les attentes respectives et les enjeux de l'étude pour chacune des parties-prenantes. On se souvient qu'au commencement de l'enquête, le commanditaire, à savoir la partie patronale du fonds de formation paritaire, se faisait le relais des attentes de la partie syndicale. La réalisation d'entretiens exploratoires a permis de préciser les choses à cet égard, en interrogeant directement l'acteur concerné. Mais ce travail de contextualisation aurait pu être mené de manière plus systématique.

Face à un commanditaire multi-partenarial représentant différentes tendances, il est également plus qu'indispensable de préciser, en début d'enquête, les modalités de restitution des résultats, ce qui n'a pu être fait à cause des difficultés déjà éprouvées pour clarifier les objectifs de l'étude. Le contenu attendu (données brutes, diagnostic, méthode quantitative, recommandations d'action, etc.) et la fréquence souhaitée ont certes été précisés mais pas la forme envisagée (exposé, présentation powerpoint, remise d'un rapport, etc.), ce qui n'a guère contribué à faciliter l'exercice. 


\section{9

\subsection{La nécessité de nouer des relations de confiance}

Comme nous l'avons souligné précédemment, la prospective métier est désormais entrée dans une nouvelle ère. Le renouvellement de méthode s'effectue entre autre via le recours à la figure de l'acteur-expert. Ce dernier doit être considéré comme « une ressource en termes d'informations rétrospectives et prévisionnelles » et comme " un producteur de représentations collectives » (Scouarnec, 2008, p.178). Il existe différents types d'experts qui ont été consultés dans le cadre de notre enquête. II s'agit de " l'expert consulté en raison de son rôle dans les processus de décision liés à la problématique » et « l'expert consulté en raison de sa connaissance directe ou indirecte du sujet, mais travaillant dans des domaines connexes mais intéressant la problématique » (Roubelat, 1999 in Scouarnec, 2008, p.178).

Si l'on se réfère à la méthodologie explicitée par Callon (1986) dans son célèbre article sur les coquilles Saint-Jacques, il semble préférable d'inclure toutes les parties prenantes concernées par le travail de problématisation. II est vrai que l'inclusion peut se faire de différentes manières (présence physique, symbolique, etc). II est cependant essentiel que tous les " actants » (Callon, 1986) soient impliqués dans la démarche d'enquête. Une fois les parties prenantes identifiées, nous avons opéré un choix entre toutes les personnes potentielles à interviewer. Les acteurs-experts ont certes été ciblés en fonction de leur proximité avec le sujet mais comment savoir s'ils ont la capacité de se mobiliser autour d'un projet et de créer du sens ? Doivent-ils posséder une fonction ou un statut particulier ? Si oui, lequel ?

Dans notre réflexion sur l'échantillonnage, nous avons eu recours à deux types d'échantillon : l'échantillon « de convenance »(Bryman et Bell, 2007) pour la phase exploratoire et l'échantillon raisonné ou « par quota » (Ghiglion et Matalon, 2004) pour la phase quantitative. Un échantillon de convenance correspond tout simplement à un échantillon qui est à la disposition du chercheur étant donné son accessibilité (Bryman et Bell, 2007). L'avantage de cette méthode est d'avoir une plus grande chance d'obtenir un taux de réponse élevé. A contrario, les résultats obtenus ne peuvent être généralisables car on ne sait pas de quelle population l'échantillon est représentatif. Notre échantillon s'est construit sur ce type d'échantillon, c'est-à-dire sur base du carnet d'adresses du commanditaire et des relations interpersonnelles existantes.

Derrière les termes "échantillon raisonné ", il faut entendre le choix d'un échantillon n'obéissant pas aux lois de l'échantillon aléatoire mais tiré sur la base de caractéristiques fixées à l'avance. Ce type d'échantillonnage a été utilisé pour définir la liste des entreprises à rencontrer dans le cadre de la phase quantitative. Nous avons eu à nouveau recours à un échantillon de convenance pour choisir les acteurs-experts à interroger. Ces derniers ont été recrutés sur base de leur expertise. 


\section{Conditions et limites d'une démarche de prospective métier dans un secteur de PME touché par la crise économique}

Au cours de l'enquête, nous remarquons combien il est difficile de mobiliser et de faire participer les acteurs à un exercice de prospective. De multiples obstacles émergent dès qu'on passe du statut de " participant symbolique " à celui de " participant physique "(Callon, 1986). Pour surmonter ces obstacles liés à la mobilisation des acteurs-experts, diverses stratégies et/ ou " dispositifs d'intéressement " (Callon, 1986) ont été mis(es) en place : l'exploitation du carnet d'adresses du fonds de formation, le recours à une technique d'enquête en face-à-face tant pour la phase exploratoire que pour la passation du questionnaire, la création de scénarii, etc. II semble nécessaire, audelà de ces dispositifs, de créer un contexte particulier de confiance pour obtenir la mobilisation des acteurs-experts.

Dans le cadre d'une enquête de type prospectif, il semble que les relations interpersonnelles jouent un rôle décisif car parler de l'avenir nécessite pour les répondants de se dévoiler. Dans quelle mesure un individu se dévoilerait-il alors que l'interrogateur est une personne « non familière » (Luhman, 2001) qui peut inspirer de la méfiance ? La confiance est liée au concept de risque car elle peut être considérée comme une source d'inquiétude en ce sens où elle consiste à accepter quelque chose qui n'est pas tout à fait sûr et à parier sur la loyauté de celui à qui I'on fait confiance (Quéré, 2001).

Nous allons nous centrer principalement sur la " confiance interpersonnelle » (Luhman, 2006). Considérée par certains auteurs comme une propriété intrinsèque des relations entre les individus (Granovetter, 1985 ; Zaheer et al., 1998 ; Quéré, 2001), elle agit comme un facteur indispensable lors de la phase de récolte des données dans une enquête. La mise en situation d'interview peut engendrer une forme d'incertitude chez le répondant. Ce dernier peut, en l'absence d'une mise en confiance, soit se taire soit adopter des comportements tels que la rétention d'informations ou, en forçant le trait, le mensonge. Le fait d'être en incertitude provoque la nécessité de développer des mécanismes de confiance entre l'intervieweur et le répondant.

Un autre élément a fortement joué en notre faveur lors de la prise de contact avec les répondants. II s'agit de " la réputation » (Orléan, 1994 ; Karpik, 1996) du commanditaire. Le fait de se référer au commanditaire lors de la prise de contact avec le répondant a augmenté les chances d'obtenir un taux de réponse élevé, d'une part, et a mis à l'aise les répondants lors de l'interview, d'autre part. La réputation agit comme un dispositif de réduction de l'incertitude et, par conséquent, de mise en confiance. 


\section{9

\subsection{Le collectif comme lieu de constitution du « praticien réflexif »}

Les résultats obtenus via le questionnaire concernant l'évolution des modes d'organisation et des pratiques de GRH - en particulier les pratiques de formation - se sont avérés quelque peu décevants dans la mesure où les changements envisagés dans les dix prochaines années restent minces. Un des premiers facteurs explicatifs peut être lié au fait que nos répondants - en majorité des responsables de PME et de TPE - ne sont guère prêts à des changements et ce, particulièrement en période de crise où ils doivent faire face à de multiples problèmes imprévus. Même si certains changements sont anticipés, ce n'est pas pour autant qu'ils se traduiront par des modes de fonctionnement nouveaux.

La théorie de la « dépendance au sentier » permet de comprendre la tendance générale des institutions à suivre un chemin qu'elles ont commencé à emprunter (Palier et Bonoli, 1999). Visiblement, le recours aux scénarii prospectifs n'a guère favorisé le dépassement d'une telle perpétuation des routines organisationnelles. Nous nous sommes en effet confrontés à une des difficultés soulevées par Gimbert (2004) lorsqu'il précise qu'un des obstacles à la mise en œuvre de la démarche prospective se réfère à la « pauvreté de l'imaginaire ». L'esprit humain a tendance à s'inscrire dans la régularité et la continuité, ce qui l'oriente naturellement vers les scénarii « classiques » d'une tendance donnée. La pauvreté de l'imaginaire s'explique, en grande partie, par une " fascination pour la raison " (Gimbert, 2004, p.87). Cette réaction est également une manière de jouer la sécurité face à un avenir incertain et face à une situation économique instable.

Un deuxième facteur explicatif du faible pourcentage d'entreprises à anticiper un changement est lié, nous semble-t-il, au manque de possibilités concrètes de « capacitation » (Sen, 2000) offertes aux répondants. La « capability » (capacité ou capabilité) repose sur cet espace des choix « réellement possibles » (Sen, 2000, 2005 ; Dupriez et al., 2007) désignant l'ensemble des opportunités dont disposent un individu, une organisation ou une institution pour réaliser ses choix d'existence (Lefèvre, 1997 ; Bonvin et Farvaque, 2008). L'approche par les capacités permet d'évaluer les situations et arrangements sociaux à partir d'un double critère : les « functionings » (fonctionnement, réalisation ou accomplissement) et l'étendue de la « liberté réelle ». L'ensemble des réalisations n'a de sens qu'associé à l'ensemble des solutions alternatives sur lesquelles l'individu et/ou l'institution peuvent se prononcer.

Dans ce cadre-ci, nous avons essentiellement travaillé sur le registre de la « capability for voice » (Bonvin et Farvaque, 2004) ou capabilité « délibérative » au sens d'Urfalino ${ }^{88}$ (2000, 2005). Tout au long de l'enquête, nous avons veillé

\footnotetext{
88. Philippe Urfalino, sociologue et politologue, directeur de recherches (CNRS) et d'études (EHESS), a principalement orienté ses recherches sur les pratiques de délibération. II n'a donc pas parlé de " capabilité délibérative » ; le concept de capabilité provenant d'Armatya Kumar Sen.
} 


\section{Conditions et limites d'une démarche de prospective métier dans un secteur de PME touché par la crise économique}

à offrir un large " éventail des possibles " aux parties prenantes : entretiens ouverts de la phase exploratoire qui ont servi à l'élaboration du questionnaire (certains en ont d'ailleurs profité pour mettre en exergue des questionnements, des incohérences ainsi que des pistes de solution) ; passation du questionnaire en face à face, ce qui a permis aux répondants d'exprimer leur point de vue sur les évolutions du secteur ; questions ouvertes permettant de faire de plus amples commentaires ; présentation des résultats de l'enquête devant les participants à l'étude, en vue de poursuivre la réflexion et d'élaborer des pistes d'action, etc. II semble cependant que cette gamme des possibles n'ait pas suffi et qu'il soit nécessaire de renforcer la capacitation des acteurs-experts afin d'accroître leur participation effective et d'activer leur imagination.

Une des forces de l'approche par les capacités est la prise en compte du concept de liberté de choix des acteurs. A tout moment, les acteurs-experts ont l'opportunité d'activer ou non leur liberté d'expression. On peut ainsi remarquer que certains répondants décident de ne pas profiter des diverses occasions pour participer et s'exprimer sur la thématique. La solution « exit » (Hirschman, 1970) s'est traduite par des comportements tels que le refus de participer à l'enquête, l'absence d'engagement, la présence de comportements passifs lors de la réalisation de l'entretien semi-directif ou de la passation du questionnaire, l'absence aux séances de restitution des résultats ou la faible interactivité lors de celles-ci, etc.

D'autres pistes sont peut-être plus appropriées pour inciter les acteurs à participer à l'étude et faire en sorte qu'ils deviennent des praticiens réflexifs (Argyris, 1995 ; Schön, 1994). Le concept de " praticiens réflexifs » est lié à celui de "savoir actionnable ». La démarche prospective peut être considérée comme un savoir « actionnable » à partir du moment où il y a présence de trois éléments : 1) une adéquation entre les intentions des acteurs et les résultats effectifs, 2) un suivi des effets sur le long terme via des missions de veille et de communication, 3) un espace de co-conception de l'action collective (Scouarnec, 2008). Nous allons voir si notre étude s'inscrit réellement dans cette optique.

Le premier élément se traduit par la rencontre d'experts permettant la réduction des asymétries d'information et, en conséquence, l'incertitude entre les différents acteurs intéressés par la problématique (Scouarnec, 2008). Tout au long de la démarche, nous avons fait appel à des personnes-ressources qui ont exprimé leurs visions du secteur étudié ainsi que leurs attentes par rapport à l'étude. L'équilibre des informations se situe, pour les acteurs de terrain, dans un échange avec les chercheurs grâce à un feed-back des résultats de l'étude.

Le deuxième élément, correspondant aux missions de veille et de communication, allie temps de réflexion et temps d'action. Les différentes phases de l'enquête (exploratoire, construction du questionnaire, analyse des résultats, feedback, 


\section{9

etc.) correspondent à l'étape de réflexion. Le moment de l'action se situe après l'enquête lors de la concrétisation des recommandations.

Le troisième élément consiste en la construction d'un espace de co-conception de l'action collective. Le terrain n'est pas à envisager comme un simple espace d'observation. L'enquête a été conçue de manière à inclure les diverses parties prenantes concernées par la problématique, dans les diverses étapes de celleci. Malgré cette volonté, la plupart des interviewés se sont montrés frileux lors de l'exercice prospectif. Suite aux résultats de l'enquête, un lieu de réflexion a tout de même été créé pour valider collectivement les pistes d'actions proposées au travers des recommandations. Cependant, vu le caractère décevant des résultats obtenus au terme de cette rencontre, il semble préférable que tous les acteurs collaborent effectivement à la production des recommandations et pas uniquement à leur validation.

L'ouverture plus large de l'éventail des possibles d'un point de vue méthodologique aurait, semble-t-il, eu pour conséquence une meilleure participation de chacun et aurait permis de pousser plus loin le processus de " capabilisation " des acteurs. Une voie envisageable pour ouvrir " l'éventail des possibles » pourrait consister en l'organisation de focus groups autour des scénarii en lieu et place des interviews individuelles. Ces collectifs d'acteurs auraient sans doute de plus grandes chances, en stimulant les interactions entre participants autour d'une thématique commune, de leur permettre de se constituer en "praticiens réflexifs » et de prendre distance par rapport à leurs systèmes de référence traditionnels. Ce collectif peut être bénéfique seulement si les acteurs sont rassurés sur le fait que l'exercice est de type prospectif et non une prévision de l'avenir.

\subsection{La méthode prospective alliée à la démarche qualitative}

Derrière les évolutions futures des métiers se pose la question de la méthodologie à utiliser où l'on retrouve d'un côté les partisans des méthodologies quantitatives et, de l'autre, les partisans des approches qualitatives. A la demande du commanditaire, notre étude s'est inscrite dans une approche à vocation plutôt quantitative : les données chiffrées sont a priori plus fiables et permettent de faire apparaître les principales tendances de manière plus difficilement réfutable. Pour le commanditaire, on écarte ainsi la crainte de généralisations abusives à partir de données peu représentatives ou de simples perceptions d'un nombre limité d'acteurs. Mais on peut se demander si cette méthodologie n'enferme pas le répondant dans une démarche trop formelle, qui, certes, aide à apprécier l'ampleur d'un phénomène mais ne permet pas d'en explorer les significations subtiles.

Prenons l'exemple de la partie du questionnaire consacrée à l'acquisition des modalités de compétences pour les métiers ouvriers. Nous y utilisons la technique du « différenciateur sémantique » (Osgood et Snider, 1969) qui permet 


\section{Conditions et limites d'une démarche de prospective métier dans un secteur de PME touché par la crise économique}

au répondant, sur un certain nombre de dimensions, de se positionner entre une série de qualificatifs opposés (formation interne/externe, courte/longue, technique/soft, etc). Une des difficultés pour lui est cependant l'absence d'échelle de mesure entre les polarités, ce qui rend l'exercice proposé très complexe. De plus, notre questionnaire l'invite à établir pour chaque dimension, la différence entre la situation actuelle - future - souhaitée pour les trois métiers ouvriers principaux présents dans l'entreprise. Au terme de l'enquête, les différences observées sont minces. Les rares souhaits exprimés relèvent avant tout de généralités (le fait de faire venir le formateur en entreprise plutôt que d'obliger le travailleur à se déplacer vers le centre de formation et ce, pour disposer des machines et outils spécifiques à l'entreprise, etc.). Sans doute disposons-nous ainsi d'indicateurs chiffrés : mais le résultat obtenu est-il pertinent ?

Friedberg (1993) insiste sur la difficulté de trouver des techniques de mesure appropriées à la nature et à la complexité des phénomènes analysés. La question que nous nous posons au terme de notre étude est donc de savoir si la méthodologie quantitative est bien appropriée à la démarche prospective poursuivie : notre propre expérience nous en a montré les limites. En outre, une démarche qualitative semble ouvrir plus facilement la voie à une certaine capacitation des acteurs, telle que nous l'avons évoquée précédemment.

II semble dès lors opportun d'allier ces deux méthodes afin de comprendre et d'expliquer les résultats obtenus. Il paraît alors important de mener à cet égard un véritable travail pédagogique pour légitimer un dispositif combinant dimension quantitative et qualitative auprès du commanditaire. Cette opération de légitimation de la méthodologie doit pouvoir s'effectuer dès le début du processus d'intervention. Elle peut faire l'objet d'intenses discussions qui sont, néanmoins, indispensables à l'élaboration d'un dispositif méthodologique adéquat en fonction du type de résultat recherché.

\section{Conclusion}

A travers cet article, nous avons discuté d'une expérience de prospective métier dans le secteur des fabrications métalliques et de l'industrie technologique, pour en dégager un certain nombre d'enseignements : l'importance du travail de contextualisation préalable, afin de préciser la nature des attentes et enjeux en présence, surtout dans le cas de commanditaires multiples ; la nécessité de nouer avec les répondants des relations de confiance pour qu'ils puissent se livrer, sans trop de risque, à l'exercice prospectif qui leur est demandé ; l'intérêt de mettre en place des lieux collectifs de débats et de confrontations en vue de transformer les répondants en " praticiens réflexifs »; le travail pédagogique à mener auprès des commanditaires en faveur d'une combinaison des approches quantitatives et qualitatives, sans doute plus adaptée à la démarche prospective. Rappelons que notre réflexion ne porte que sur un seul cas, réalisé dans une 


\section{9 \\ management \& avenir}

région et un secteur d'activité (les fabrications métalliques) lourdement touchés par la crise économique de 2009. Elle est donc fortement marquée par les spécificités de ce contexte. Les résultats ne se référent qu'au secteur étudié et ne peuvent être extrapolés à d'autres car chacun d'entre eux possède ses caractéristiques propres. D'un point de vue méthodologique, il est évident que d'autres expériences du même type devraient être analysées dans une perspective comparative, en vue d'enrichir les enseignements que nous en avons dégagés. Certains de ceux-ci devraient s'avérer pertinents dans la majorité des cas, d'autres - tels que l'échange collectif autour des scénarii ou la préférence donnée à l'approche qualitative - demanderaient à être étayés de manière plus rigoureuse. II n'empêche que cette démarche, moyennant quelques adaptations, peut être généralisée à d'autres secteurs ou métiers - comme le secteur de la location ou les métiers employés - ce qui se fait actuellement en Belgique.

\section{Bibliographie}

Argyris C. (1995), Un savoir pour agir. Surmonter les obstacles à l'apprentissage organisationnel, InterEditions, Paris.

Bellini S. (2009), "Les « chemins de GPEC ». Les enseignements d'une recherche Intervention sur l'appropriation de la démarche dans un secteur associatif », Adresse URL : http://www.reims-ms.fr/agrh/docs/actes-agrh/pdf-des-actes/2009bellini014.pdf

Berger G. (1967), Les étapes de la prospective, PUF, Paris.

Berger G. (1964), Phénoménologie du temps et Prospective, PUF, Paris.

Bonvin J.-M. et Farvaque N. (2008), Amartya Sen. Une politique de la liberté, Michalon, Paris.

Bonvin J.-M. et Farvaque N. (2004), « Capability for work and capability for voice », Kluwer Acadmic Publishers, Pays-bas.

Boyer L. et Scouarnec A. (2008), « Des « possibles » environnementaux aux « possibles » organisationnels : quelle prospective métiers de la publicité et de la communication », Revue Management \& Avenir, No. 15, p. 104-119.

Boyer L. et Scouarnec A. (2002), " La prospective métier : définition, concept et méthodologie », Cahier de recherche DMSP.

Bryman A. et Bell E. (2007), Business research methods, Oxford University Press, NewYork.

Callon M. (1986), « Eléments pour une sociologie de la traduction », Année sociologique, Vol. XXXVI, p. 169-208.

De Jouvenel F. (2009), « La prospective des territoires urbains sensibles : la construction de scénarios, et quelques autres méthodes », Futuribles, France.

De Jouvenel H. (1999), "La démarche prospective. Un bref guide méthodologique ", Revue Futuribles, No. 247, p. 47-68.

Dupriez V., Orianne J.-F. et Verhoeven M. (2007), « Vers des politiques d'éducation « capabilisantes »? Une analyse critique de l'action publique en matière d'éducation », Formation Emploi, No. 98, p. 93-107.

Friedberg E. (1993), Le pouvoir et la règle. Dynamiques de l'action organisée, Seuil, Col. Sociologie, Paris.

Ghiglion R. et Matalon B. (2004), Les enquêtes sociologiques. Théories et pratique, Armand Colin, Paris.

Gilbert P. (2006), La gestion prévisionnelle des Ressources Humaines, La découverte, Paris.

Gimbert L. (2004), « La Prospective : pour un retour aux fondamentaux » in Revue 


\section{Conditions et limites d'une démarche de prospective métier dans un secteur de PME touché par la crise économique}

Management \& Avenir, No. 2, p. 83-95.

Gonod P. (1996), « Dynamique des systèmes et méthodes prospective », in Travaux et recherches de prospective, Futuribles, No. 2.

Granovetter M. (1985), "Economic action and social structure the problem of embeddedness ", American Journal of Sociology, Vol. 91, No. 3, p. 481-510.

Hirschman A.-O. (1970), Exit, Voice, Loyalty, Responses to Decline in Firms, Organizations, and States, Harvard University Press.

Julien P.-A., Lamonde P. et Latouche D. (1975), " La méthode des scénarios en prospective », L'actualité économique, Vol. 51, No. 2, p. 253-281.

Karpik L. (1996), "Dispositifs de confiance et engagements crédibles », Sociologie du travail, Vol. 38 , No. 4, p. 527-550.

Lefèvre C. (1997), « Un modèle multidimensionnel de la qualité de vie : Analyse des bénéfices des entreprises de formation par le travail en Hainaut ", Les Cahiers du CERISIS, No. 3b.

Luhmann N. (2006), La confiance : un mécanisme de réduction de la complexité sociale, Economica, Paris.

Luhmann N. (2001), "Confiance et familiarité. Problèmes et alternatives », Réseaux, Vol. 19, No. 108 , p. $15-35$.

Noguera F. et Lartigau J. (2009), « De la prospective à la gestion prévisionnelle des métiers et des compétences dans la fonction publique hospitalière : enjeux et perspectives ", Revue Management \& Avenir, No. 25, p. 290-314.

Orléan A. (1994), " Sur le rôle respectif de la confiance et de l'intérêt dans la constitution de l'ordre marchand ", Revue du MAUSS, No. 4, p. 17-36.

Osgood C.-E. et Snider J.-G. (1969), Semantic Differential Technique: A Sourcebook, Aldine, Chicago.

Palier B. et Bonoli G. (1999), " Phénomènes de path dependence et réformes des systèmes de protection sociale ", Revue française de science politique, Vol. 49, No. 3, p. 399-420.

Pichault F. (2005), «L'intervention en organisation : une question de légitimité systémique », RIP, No. 26, p. 63-96.

Quéré L. (2001), « La structure cognitive et normative de la confiance », Réseaux, Vol. 19, No. 108 , p. $125-152$.

Schön D. (1994), Le praticien réflexif. À la recherche du savoir caché dans l'agir professionnel, Éditions Logiques, Montréal.

Scouarnec A. (2008), "Plaidoyer pour un "renouveau " de la prospective ", Revue Management \& Avenir, No. 19, p. 171-186.

Scouarnec A. et Yanat Z. (2003), « La prospective métier : un instrument au service d'une GRH innovante », Adresse URL : http://www.reims-ms.fr/agrh/docs/actes-agrh/pdf-desactes/2003scouarne-yanat098.pdf

Sen A. (2005), Rationalité et liberté en économie, Odile Jacob, France.

Sen A. (2000), Repenser l'inégalité, Seuil, France.

Thamain J.-L. (2009), « Anticiper en GRH, prévision et prospective, un diagnostic des pratiques et des représentations ", Revue Management \& Avenir, No. 25, p. 272-289.

Urfalino P. (2005), « La délibération n'est pas une conversation. Délibération, décision collective et négociation », Négociations, No. 2, p. 99-114.

Urfalino P. (2000), « La délibération et la dimension normative de la décision collective » in Commaille J., Dumoulin L. et Robert C., La juridicisation du politique, L.G.D.J., France, p. 165-193.

Zaheer A., McEvily B. et Perrone V. (1998), « Does Trust Matter? Exploring the Effects on Inter-organizational and Interpersonal Trust on Performance », Organizational Science, Vol. 9, No. 2, p. 141-159. 